Bio - grafia. Escritos sobre la Biología y su Enseñanza. ISSN 2027

Edición Extraordinaria. p.p. 1090- 1101

Memorias del VIII Encuentro Nacional de Experiencias en Enseñanza de la Biología y la Educación Ambiental. III Congreso Nacional de Investigación en Enseñanza de la Biología.

\title{
EL CONOCIMIENTO DIDÁCTICO SOBRE LA CÉLULA Y EL ANÁLISIS CRÍTICO DE LAS PROPUESTA (ORIENTACIONES) DE ENSEÑANZA DE DOS PROFESIONALES DEL ÁREA BIOLÓGICA QUE EJERCEN EN LA EDUCACIÓN SECUNDARIA
}

\author{
YAÑEZ OLIVA, RUTH ${ }^{1}$ \\ TAPIA SALAZAR, GUSTAVO
}

\section{RESUMEN}

B

I

0

P

0

N

E

N

c

I

A

a

MARCO TEÓRICO

\footnotetext{
${ }^{1}$ Universidad Central de Chile

${ }^{2}$ Member The Critical Thinking Community

${ }^{1}$ ruth.yanez@ucentral.cl

${ }^{2}$ tavociencias@gmail.com
}

La enseñanza, aprendizaje y evaluación de la ciencia que se enseña para su aprendizaje, es, de permanente interés. De la misma forma lo es, el análisis crítico que se debiera realizar sobre el hacer dentro de un contexto y el conocimento -profesional- que declara y explicita el profesorado en la enseñanza y evaluación para el aprendizaje resultando de interés explorar, el conocimiento didáctico y su análisis crítico a la hora de intencionar y proponer u orientar la enseñanza. El objetivo de este trabajo es: Caracterizar el conocimiento didáctico, desde una mirada crítica de las orientaciones de la enseñanza de profesionales del área biológica, sin formación pedagógica, que ejercen -como profesores- en la educación secundaria través de un cuestionario CoRe aplicado a los profesores. y la propuesta (test) de razonamiento de Paul y Elder (1997). Los resultados revelan que los propósitos de enseñanza y aprendizaje que declaran estos profesores son lograr la comprensión de la célula y el desarrollo de habilidades científicas. No obstante, éstos no consideran la naturaleza evolutiva del conocimiento científico. Ni tampoco es posible identificar con exactitud los elementos del razonamiento que siguen los profesores para orientar la enseñanza.

Palabras clave: CoRe, conocimiento didáctico, célula, profesionales de área biológica, pensamiento crítico. 
Bio - grafia. Escritos sobre la Biología y su Enseñanza. ISSN 2027

Edición Extraordinaria. p.p. 1090- 1101

Memorias del VIII Encuentro Nacional de Experiencias en Enseñanza de la Biología y la Educación Ambiental. III Congreso Nacional de Investigación en Enseñanza de la Biología.

La enseñanza que promueve un profesor/a en la educación primaria, secundaria y universitaria, es de permanente interés para la política pública, la comunidad científica y la sociedad, porque busca lograr aprendizaje -de calidad- $y$, son estos los que reportan, de alguna manera, la eficacia del profesorado. Esta es, una mirada restringida y limitada del proceso de enseñanza y aprendizaje, pero no se puede desconocer que es un núcleo de interés. Como lo es, el protagonismo que deben adquirir los estudiantes, a la hora de exigir contenidos con sentido y valor para sus vidas y desarrollo personal. Por tanto, el profesorado debe movilizar su conocimientoo profesional para atender a dichas exigencias. Entendemos este omo un conjunto de ideas integradas por cuatro componentes: saber académico, teorías implícitas, saber basado en la experiencia y rutinas y guiones (Porlán et al., 1997; Perafan, 2005) tiene un carácter pragmático y que se construye en contextos donde se ejerce la práctica (Rodrigo et al., 1993) por lo tanto es una reconstrucción derivada de la experiencia (Elliot, 1999; Marrero, 1993) que se conecta con el conocimiento teórico adquirido previamente (Angulo, 1999) y que finalmente, corresponde a un conocimiento transformado producto de la interacción de las cuatro ideas antes planteadas. Es la pre-condición para el éxito de la enseñanza (Fisher et al., 2012) que implica cinco núcleos de interés: a) el profesor/a y su compromiso con los estudiantes y su aprendizaje; b) el conocimiento de la materia y cómo enseñarla a sus estudiantes; c) la responsabilidad en el monitoreo del aprendizaje de los estudiantes; d) reflexión sistemática sobre su práctica y aprendizaje desde la experiencia; y e) miembro de una comunidad de aprendizaje (grupo de profesores). De la misma forma, el conocimiento profesional es dividido en siete categorías según Shulman (1986, 1987). Creemos que aproximarnos al conocimiento profesional $y$, en particular, al conocimiento didáctico del contenido (CDC) es una oportunidad y necesidad, dado que en Chile son escasos los trabajos sobre esta temática en profesores de biología u otros profesionales que ejercen la docencia.

Por otra parte, para analizar desde una mirada crítica el pensamiento del profesor respecto del conocimiento didáctico del contenido es necesario situarnos en el pensamiento crítico su definición y su enseñanza. Sin embargo, no existe concenso a la hora de definir el pensamiento crítico pero, si y se a logrado tomar acuerdos de manera uniforme respecto de su definición dada la amplitud de los campos de estudios que lo abordan, esto es, filosóficos, cognitivos, sicológicos, entre otros. Los que, coinciden en que se debe seguir investigando sobre las estrategias para el desarrollo de habilidades de pensamiento crítico y sus disposiciones al aprendizaje. Además, existe una gran variedad de enfoques y modelos 
Bio - grafia. Escritos sobre la Biología y su Enseñanza. ISSN 2027

Edición Extraordinaria. p.p. 1090- 1101

Memorias del VIII Encuentro Nacional de Experiencias en Enseñanza de la Biología y la Educación Ambiental. III Congreso Nacional de Investigación en Enseñanza de la Biología.

de enseñanza del pensamiento crítico, pero pocos de ellos han sido probados empíricamente, lo que acentúa la escasez de consenso sobre la definición del pensamiento crítico y también ha obstaculizado en los esfuerzos por desarrollar instrumentos para desarrollar y evaluar el pensamiento crítico (Ennis,1992; Halpern, 1993; Paul, 1993; Perkins y Salomon, 1989). En esta misma línea algunas visiones sostienen que los estudiantes desarrollan habilidades de pensamiento crítico sólo por el hecho de asistir a clases, escuchar conferencias y participar en las discusiones en clase, o bien rindiendo exámenes o evaluaciones. No obstante, numerosos estudios, han indicado que la mejora del pensamiento de los estudiantes requiere una enseñanza más explícita e integradora de las habilidades de pensamiento crítico (Bangert-ahoga y Bankert, 1990; Halpern, 1998; Keeley, Browne, y Kreutzer, 1982; Perkins, 1989; Quellmalz, 1989; Underbakke, Borg, y Peterson, 1993).

Lo anterior nos permite destacar la implicancia sobre el aprendizaje cuando los estudiantes piensan de una manera activa sobre lo que aprenden y a su vez demanda de sus profesores un análisis reflexivo previo, razonado y responsable sobre qué, por qué y/o cómo el profesor, enseñará un determinado tópico. En nuestro estudio, la célula.

Paul $(2002,2007,2015)$ afirma que estas definiciones enfatizan en el aspecto metacognitivo del pensamiento crítico, el pensamiento independiente, y la importancia de aprender a evaluar el pensamiento (propio o de otra persona) de acuerdo a estándares normativos. Para él pensar críticamente, es un medio para combatir la influencia de los prejuicios, que influyen al emitir un juicio sobre un problema. Este autor ha desarrollado un concepto integrador del pensamiento crítico, que se basa en ideas a partir de una variedad de campos y perspectivas argumentando que el pensamiento crítico requiere una integración de los dominios cognitivos y afectivos, presentes en cualquier disciplina, que debe ser visto y enseñado como modo de pensamiento (es decir, la historia como pensamiento histórico, la biología como pensamiento biológico, entre otros), y su modelo de forma crítica. Dado que lo concibe como un proceso intelectualmente disciplinado

de conceptualizar, aplicar, analizar, sintetizar y/o evaluar información recopilada o generada por observación, experiencia, reflexión, razonamiento o comunicación, de manera activa y hábil,como una guía hacia la creencia y la acción. Incluye

elementos cognitivos del razonamiento, estándares normativos y disposiciones afectivas (Fundación para el Pensamiento Crítico, 1996). que conducen al análisis de tres dimensiones cruciales: a.- las perfecciones del pensamiento, b.- Los elementos del pensamiento y c.- los dominios. Así, el pensamiento crítico es un pensamiento disciplinado, autodirigido, que ejemplifica las perfecciones del pensamiento apropiado a un modo particular (en una 
Bio - grafia. Escritos sobre la Biología y su Enseñanza. ISSN 2027

Edición Extraordinaria. p.p. 1090- 1101

Memorias del VIII Encuentro Nacional de Experiencias en Enseñanza de la Biología y la Educación Ambiental. III Congreso Nacional de Investigación en Enseñanza de la Biología.

disciplina por ejemplo) o un dominio del mismo. Esto demanda en el pensamiento del profesor tanto estándares intelectuales como virtudes intelectuales que deben estar articuladas cuando el profesor se piensa o intenciona la enseñanza, dado que de esta forma le ayudarán en la superación de los prejuicios y suposiciones infundadas que muchas veces se transfieren al problema que se quiere resolver, a la hora de planear la enseñanza. Esto fortalece la idea de instalar en el pensamiento del profesor, el análisis crítico (o pensamiento crítico) en el centro de su práctica pedagógica que se inicia cuando intenciona o propone la enseñanza.

Paul (1997) entrega una definición que conduce al análisis de tres dimensiones cruciales: Las perfecciones del pensamiento, Los elementos del pensamiento y Los dominios. Así, el pensamiento crítico es un pensamiento disciplinado, autodirigido, que ejemplifica las perfecciones del pensamiento apropiado a un modo particular (en una disciplina por ejemplo) o un dominio del mismo.

\section{PENSAMIENTO CRÍTICO Y ENSEÑANZA}

La enseñanza asociada a un análisis crítico, demanda la comprensión de los conceptos y principios en el pensamiento crítico y la aplicación de estos conceptos a través del currículo (Paul y Elder, 2010). Significa desarrollar estrategias poderosas que surgen cuando empezamos a entender el pensamiento crítico e involucrarlo desde el momento en que se intenciona o propone la enseñanza. Diseñando, por ejemplo, estrategias o métodos de enseñanza que involucren activamente a los estudiantes en su proceso de aprendizaje. No obstante, el profesor tiene una gran responsabilidad, cuando el profesor toma decisiones asociadas a la enseñanza, él está garantizando que los estudiantes aprenderán a disciplinar su forma de pensar, ya que esta propuesta de enseñanza les permitirá a los estudiantes razonar a través de contenidos. Lo que implica que ellos, por ejemplo, aprenderán la importancia de utilizar los principios del pensamiento crítico en el razonamiento a través de los problemas y cuestiones en todos los temas que el profesor propone. Lo anterior, sólo es posible si el profesor ha sido capaz de reflexionar críticamente sobre los elementos esenciales de su propio pensamiento a la hora de intencionar la enseñanza. El presente 
Bio - grafia. Escritos sobre la Biología y su Enseñanza. ISSN 2027

Edición Extraordinaria. p.p. 1090- 1101

Memorias del VIII Encuentro Nacional de Experiencias en Enseñanza de la Biología y la Educación Ambiental. III Congreso Nacional de Investigación en Enseñanza de la Biología.

estudio se adscribe a la definición de pensamiento crítico de los autores Paul y Elder (2007) y a la propuesta de desarrollo que ellos proponen. En este caso, partiendo desde el análisis del pensamiento del profesor cuando orienta la enseñanza de un contenido o tópico particular, en nuestro caso la célula.

\section{METODOLOGÍA}

Se identifican a 2 profesionales del área biológica que ejercen como profesores de biología en centros de educación pública (municipal y subvencionada). Cada uno de los participantes, además, está en programa de formación de profesores para obtener el título de profesor de biología. Los participantes son un Ingeniero en Biotecnología, un médico veterinario, 3 años de experiencia laboral en aula.

Cuestionario sobre la representación del contenido de la célula (CORE)

Para documentar el CDC de los participantes se usó un cuestionario escrito conocido como Representación del Contenido -CoRe de "Content Representation"- propuesto por Loughran et al., (2003)) El cuestionario está constituido por dos (2) secciones como se muestra en la tabla 1 del Anexo.

Tabla 1. Preguntas del CoRe según tres dimensiones del CDC

\begin{tabular}{|l|l|}
\hline $\begin{array}{c}\text { Dominio del Conocimiento } \\
\text { Didáctico }\end{array}$ & \multicolumn{1}{c|}{ Preguntas del ReCo } \\
\hline \multirow{4}{*}{$\begin{array}{l}\text { Orientación hacia la } \\
\text { enseñanza de la célula } \\
\text { (OEC) }\end{array}$} & $\begin{array}{l}\text { 1. ¿Qué espera que los/las estudiantes aprendan } \\
\text { cuando usted enseña el contenido de la célula? }\end{array}$ \\
\cline { 2 - 2 } & $\begin{array}{l}\text { 2. ¿Por qué es importante que los estudiantes aprendan } \\
\text { este conocimientos -la célula- (JUSTIFICACIÓN) }\end{array}$ \\
\cline { 2 - 3 } & $\begin{array}{l}\text { 3. ¿Cuál es el rol del profesor durante el proceso } \\
\text { enseñanza-aprendizaje y evaluación de la Célula? }\end{array}$ \\
\cline { 2 - 2 } & 4. ¿Cuál es el rol del estudiante durante el proceso \\
\hline
\end{tabular}


Bio - grafia. Escritos sobre la Biología y su Enseñanza. ISSN 2027

Edición Extraordinaria. p.p. 1090- 1101

Memorias del VIII Encuentro Nacional de Experiencias en Enseñanza de la Biología y la Educación Ambiental. III Congreso Nacional de Investigación en Enseñanza de la Biología.

\begin{tabular}{|c|c|}
\hline & enseñanza-aprendizaje y evaluación de la Célula? \\
\hline \multirow{3}{*}{$\begin{array}{ll}\text { Conocimiento sobre el } \\
\text { Entendimientos } & \text { de } \\
\text { Estudiantes (CEE) } & \end{array}$} & $\begin{array}{l}\text { 5. ¿Cuál (es) es (son) el/los principal(es) errores que } \\
\text { comenten los estudiantes durante el aprendizaje de } \\
\text { la célula }\end{array}$ \\
\hline & $\begin{array}{l}\text { 6. ¿Qué dificultades o limitaciones presentan los } \\
\text { estudiantes para el aprendizaje de la célula? }\end{array}$ \\
\hline & $\begin{array}{l}\text { 7. Como profesor/a, ¿qué sabe acerca de las ideas } \\
\text { previas de los estudiantes sobre la célula? }\end{array}$ \\
\hline \multirow[t]{5}{*}{$\begin{array}{l}\text { Conocimiento sobre } \\
\text { Instrucción, Estrategias y } \\
\text { Representación (CIER) }\end{array}$} & $\begin{array}{l}\text { 8. ¿Cuál es la secuencia de enseñanza que usa } \\
\text { habitualmente para la enseñanza de la célula? } \\
\text { Describa }\end{array}$ \\
\hline & $\begin{array}{l}\text { 9. Como profesor, ¿qué dificultades o limitaciones } \\
\text { presenta cuando enseña el contenido de la célula? }\end{array}$ \\
\hline & $\begin{array}{l}\text { 10. ¿Existe algún otro factor que influya en la enseñanza } \\
\text { sobre la célula? }\end{array}$ \\
\hline & $\begin{array}{l}\text { 11. ¿Qué estrategias se podrían usar para enseñar } \\
\text { sobre la célula? ¿por qué? }\end{array}$ \\
\hline & $\begin{array}{l}\text { 12. ¿Cómo se enseña el contenido de la célula en el } \\
\text { contexto escolar? ¿Haciendo qué? }\end{array}$ \\
\hline $\begin{array}{l}\text { Conocimiento } \\
\text { Evaluación (CEV) }\end{array}$ & $\begin{array}{l}\text { 13. Como profesor, ¿cómo podría evaluar el nivel de } \\
\text { comprensión o el nivel de confusión de los } \\
\text { estudiantes sobre la célula? }\end{array}$ \\
\hline
\end{tabular}

\section{Análisis crítico de lo que declara el profesor}

Para el análisis crítico de lo que declara el profesor cuando orienta la enseñanza, se utiliza una prueba con base en la propuesta de razonamiento de Paul y Elder (1997) donde describen ocho elementos del razonamiento (Figura 1, Figura 2), que nos permite evaluar el pensamiento crítico del profesor cuando orienta la enseñanza a través de la evaluación de estándares intelectuales. Dado que para enseñar una materia de un modo crítico se requiere que los estudiantes se apropien de las estructuras intelectuales básicas de la disciplina (los elementos del pensamiento figura1). 
Bio - grafia. Escritos sobre la Biología y su Enseñanza. ISSN 2027

Edición Extraordinaria. p.p. 1090- 1101

Memorias del VIII Encuentro Nacional de Experiencias en Enseñanza de la Biología y la Educación Ambiental. III Congreso Nacional de Investigación en Enseñanza de la Biología.

\section{FIGURA 1: ELEMENTOS DEL RAZONAMIENTO FIGURA 2: PASOS DEL DESARROLLO DEL PENSAMIENTO CRÍTICO}
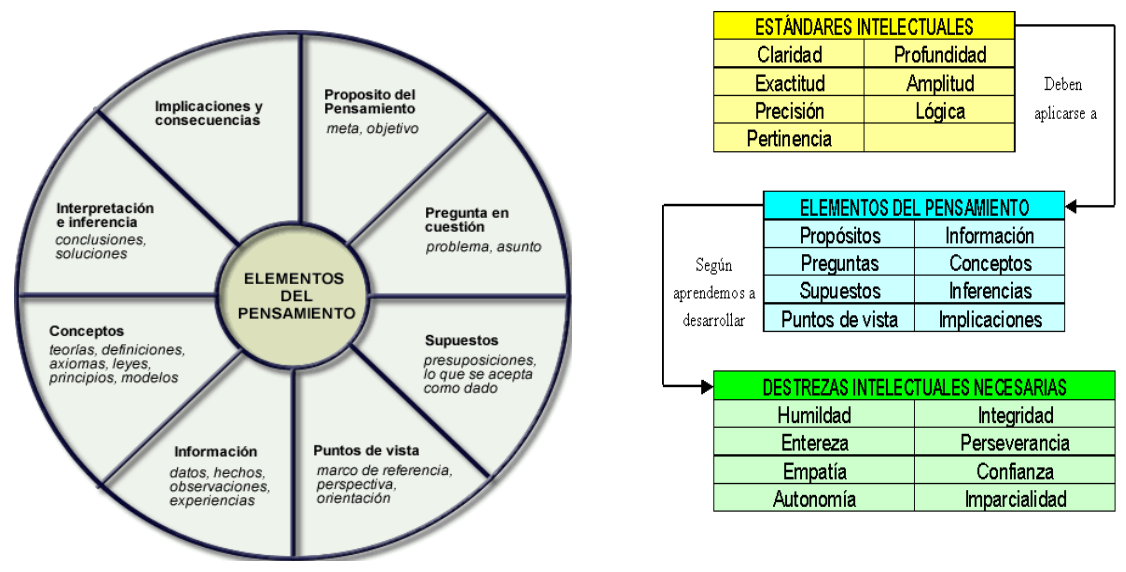

(Extraido de: http://sickmind.com.ar/blog/wpcontent/uploads/2010/10/4.png)

\section{RESULTADOS}

En relación a la dimensión Orientación para la enseñanza de la célula, los profesores entrevistados dan cuenta de lo siguiente:

Para Mario (Ingeniero) velar por cumplir con los contenidos mínimos obligatorios es fundamental, además que incluir los contenidos conceptuales, procedimentales y actitudinales, es importante. Dado que así los estudiantes pueden adquirir los contenidos y desarrollar las habilidades relacionadas con el tema y nivel que corresponde. Además, que estos contenidos están dados por los programas de estudio, y se integran con las habilidades de pensamiento científico referentes al análisis de investigaciones clásicas relacionadas con moléculas que participan en el metabolismo. Se propone desarrollar habilidades que le permitan al estudiante organizar, interpretar datos, formular explicaciones y conclusiones. Este profesor sostiene que el desarrollo de habilidades debe 
Bio - grafia. Escritos sobre la Biología y su Enseñanza. ISSN 2027

Edición Extraordinaria. p.p. 1090- 1101

Memorias del VIII Encuentro Nacional de Experiencias en Enseñanza de la Biología y la Educación Ambiental. III Congreso Nacional de Investigación en Enseñanza de la Biología.

apoyarse en las teorías y conceptos científicos sobre la composición y fisiología celular. Dado que es fundamental que los estudiantes adquieran conocimiento de la célula, porque este conocimiento, constituye el inicio del estudio de las funciones vitales de los organismos. Así mismo, amplía el vocabulario de los estudiantes y los introduce en el lenguaje científico que comúnmente se usa en la investigación científica.

Por otra parte, cuando enseña el contenido de la célula considera necesario abarcar todos los estilos de aprendizaje, porque así es posible garantizar aprendizajes significativos en todos los estudiantes. Por eso, comienza indagando para determinar el estilo -de aprendizaje- del estudiante, luego diseña actividades didácticas de acuerdo a éstos para que así promuevan el desarrollo de habilidades, tales como: investigación de estudios clásicos, clasificación y análisis de datos.

Por su parte cuando Clara(Médico veterinario) es consultada sobre cómo orienta la enseñanza de la célula ella sostiene que espera que los estudiantes comprendan que la célula forma nuestro cuerpo, y que la célula es la "maquinaria" (hacen algo/todo) que permite la vida. Lo que considera importante porque así, los estudiantes, podrán comprender como funciona su cuerpo y lograrán llevar -el conocimiento de la célula- a la cotidianidad. Así también entenderán cómo se producen ciertas enfermedades de importancia para los seres vivos. Para enseñar este contenido en el contexto escolar ella lo que hace es exponer los contenidos, observar células en el laboratorio y "realizar" maquetas con sus estudiantes.

En términos generales respecto a la dimensión Orientación para la enseñanza de la célula, los profesores entrevistados cuando enseñan el contenido de la célula esperan que los/las estudiantes aprendan -comprendan- algunos contenidos teóricos específicos que les permita ser capaces de relacionar la estructura y la función de algunos tejidos, así como también desarrollen habilidades de pensamiento científico como análisis de investigaciones $e$ interpretación de datos, otros esperan que desarrollen habilidades cognitivas como comprender, reconocer o conocer un determinado contenido. Los profesores sólo manifiestan la intención de que aprendan contenidos actitudinales y afectivos, indicando Mario que esto les permitirá "... comprender su cuerpo, aceptarlo y amarlo". Además, consideran importante que los estudiantes aprendan y conozcan la célula porque le atribuyen un rol fundamental en lo que se refiere a la comprensión del funcionamiento del cuerpo y cómo éste incide en las relaciones que se dan entre organismos. Asimismo, les permitirá comprender las teorías que explican la fisiología celular y sustentan el buen funcionamiento de un individuo, así como la causa de enfermedades. De la misma forma, el 
Bio - grafia. Escritos sobre la Biología y su Enseñanza. ISSN 2027

Edición Extraordinaria. p.p. 1090- 1101

Memorias del VIII Encuentro Nacional de Experiencias en Enseñanza de la Biología y la Educación Ambiental. III Congreso Nacional de Investigación en Enseñanza de la Biología.

conocimiento de las relaciones de complejidad de organización biológica les permitiría comprender el proceso evolutivo

En lo que refiere a la mirada crítica de la Orientación para la enseñanza de la célula, por parte de cada profesor:

Al contrastar los resultados con lo que plantea Paul y Elder (2004), pareciera que los profesores de este estudio, al intencionar los propósitos de enseñanza no son capaces de reconocer los elementos del pensamiento que se ponen en juego $y$, que definen el razonamiento del pensamiento. Lo que permitirá analizarlo, cuando se trata de comprender por qué el profesor orienta la enseñanza de una forma particular. A decir:

Cuando Mario toma decisiones respecto de los propósitos, él sostiene que es fundamental "cumplir con los contenidos mínimos obligatorios, además de incluir los contenidos conceptuales, procedimentales y actitudinales. Porque es de esta manera como los estudiantes podrán adquirir los contenidos y desarrollar las habilidades relacionadas con el tema y nivel que corresponde..." Desconociendo que cuando un profesor se "piensa" la enseñanza debe existir un propósito con un punto de vista, que está basado en suposiciones por parte del profesor que conducirán a implicaciones y consecuencias que facilitarán el aprendizaje de sus estudiantes. Para lo que se deberá usar conceptos, ideas y teorías que permitirán a los estudiantes, interpretar datos, hechos y experiencias para dar solución a problemas. Según lo que Mario plantea, sus propósitos tienen un claro objetivo. No obstante, pareciera que para garantizar un propósito de enseñanza exitoso, deberían estar presente otros elementos del pensamiento, cuando él intenciona (piensa) y orienta la enseñanza. Tales como, ¿Qué preguntas clave trato de contestar? ¿Por qué es importante que los estudiantes aprendan este contenido?, ¿Cuáles son los conceptos esenciales asociados a este contenido?, ¿Para qué le servirá al estudiante aprender este contenido?, ¿Qué inferencias o conclusiones podrá plantear?, El estudiante, ¿Podrá plantear nuevas preguntas?., entre otras.

Al globalizar el pensamiento de este profesor, con el instrumento utilizado: falta claridad, profundidad, por ejemplo cuando quiere que los estudiantes desarrollen la capacidad de ver relaciones y de apreciar las implicaciones de esas relaciones en la determinación de la solución del problema, careciendo también de significado y lógica, principalmente.

Por su parte, Clara al orientar la enseñanza de la célula espera que los estudiantes comprendan que la célula forma nuestro cuerpo, y que la célula es la "maquinaria" (hacen algo/todo) que permite la vida. Dando cuenta que también tiene un propósito claro, que 
Bio - grafia. Escritos sobre la Biología y su Enseñanza. ISSN 2027

Edición Extraordinaria. p.p. 1090- 1101

Memorias del VIII Encuentro Nacional de Experiencias en Enseñanza de la Biología y la Educación Ambiental. III Congreso Nacional de Investigación en Enseñanza de la Biología.

corresponde a que los estudiantes comprendan dado que esto les permitirá a los comprender como funciona su cuerpo y podrán llevar -el conocimiento de la célula- a la cotidianeidad, manifestándose aquí las implicaciones y consecuencias del propósito de enseñanza. No obstante, no da, cuenta de la relevancia de utilizar tópicos o conceptos que ayudarán a contestar preguntas y/o resolver problemas del entorno inmediato o cotidiano. Aún cuando es el profesor quientrabajar con conocimientos y con procesos de pensamiento, que debieran diferenciarse y clasificarse. Dando cuenta de ausencia de precisión, imparcialidad, amplitud y lógica, que en nada favorece en el estudiante la adquisición de elementos de juicio claros y útiles para desarrollar con éxito el pensamiento crítico en sus contextos cotidianos

\section{CONCLUSIONES}

Los profesores formulan sus objetivos y propósitos de enseñanza sin considerar cómo se estructura, evoluciona y produce el conocimiento científico. Lo que indica, desde la mirada crítica, que los profesores de este estudio intencionan una enseñanza para el aprendizaje, sin embargo, al no considerar criterios intelectuales que permitan juzgar su razonamiento a la hora de proponer un tópico específico de aprendizaje, se sesgan sus decisiones a la hora de tomar decisiones sobre la enseñanza. Esto es, cuando se analiza el pensamiento del ámbito del conocimiento didáctico del contenido, orientación a la enseñanza, éste presenta algunos criterios intelectuales claros según lo que propone Elder y Paul (2007), como por ejemplo dar respuesta a un propósito, principalmente y en algunos dar respuesta a las implicancias y algunas respuestas asociadas a la relevancia de aprender algunos conceptos que permitirán comprender el tópico célula. No obstante, en su mayoría, están ausentes aún cuando son necesarios para evaluar si en el pensamiento del profesor hay un análisis crítico cuando propone la enseñanza. Lo que da cuenta que el razonamiento del profesor para planear una enseñanza crítica, carece de elementos sustanciales que permitirán lograr aprendizajes profundos $y$, con sentido para el estudiante. Lo que hace necesario y urgente profundizar y trabajar en este campo del conocimiento. Por otra parte, al situarse desde 
Bio - grafia. Escritos sobre la Biología y su Enseñanza. ISSN 2027

Edición Extraordinaria. p.p. 1090- 1101

Memorias del VIII Encuentro Nacional de Experiencias en Enseñanza de la Biología y la Educación Ambiental. III Congreso Nacional de Investigación en Enseñanza de la Biología.

las estrategias que se utilizan para enseñar, éstas son deficientes y permiten que los estudiantes aborden pobremente los análisis de problemas científicos lo que les impide tomar conciencia que no están aprendiendo. .

\section{REFERENCIAS BIBLIOGRÁFICAS}

ALVARADO, C. y GARRITZ, A. (2012). Un acercamiento al conocimiento didáctico de acidez y basicidad, de profesores mexicanos de Bachillerato y Licenciatura, $X$ Congreso Nacional de Investigación Educativa.

BANGERT-DROWNS, R. L., \& BANKERT, E. (1990, April). Meta-analysis of effects of explicit instruction for critical thinking. Paper presented at the meeting of the American Educational Research Association, Boston, MA. (ERIC Document Reproduction Service No. ED 328 614)

CARTER, K. (1990). Teachers' knowledge and learning to teach. En R. Houston (ed.), Handbook of Research on Teacher Education. Nueva York: Mac Millam, pp.291-310.

ELDER, L. y PAUL RICHARD (2007). Critical Thinking Competency Standards. Standards, Principles, Performance Indicators, and Outcomes With a Critical Thinking Master Rubric, the foundation for critical thinking. Tomales pp.56.

HALPERN, D. F. (1998). Teaching critical thinking for transfer across domains. American Psychologist, 53 (4), 449-455.

KEELEY, S. M., BROWNE, M. N., \& KREUTZER, J. S. (1982). A comparison of freshmen and seniors on general and specific essay tests of critical thinking.

PERKINS, D. N. (1989). Reasoning as it is and could be: An empirical perspective. In D. M. Topping, D. S. Cromwell, \& V. N. Kobayaski (Eds.), Thinking across cultures: Third international conference on thinking (175-194). Hillsdale, NJ: Erlbaum.

QUELLMALZ, E. S. (1987). In J. B. BARON \& R. J. STERNBERG (Eds.), Teaching thinking skills: Theory and practice (pp. 86-105). New York: W. H. Freeman. 
Bio - grafia. Escritos sobre la Biología y su Enseñanza. ISSN 2027

Edición Extraordinaria. p.p. 1090- 1101

Memorias del VIII Encuentro Nacional de Experiencias en Enseñanza de la Biología y la Educación Ambiental. III Congreso Nacional de Investigación en Enseñanza de la Biología.

UNDERBAKKE, M., BORG, J. M., \& PETERSON, D. (1993). Researching and developing the knowledge base for teaching higher order thinking. Theory into Practice, 32 (3), 138-146.

PAUL, R., \& ELDER, L. (1997). Critical thinking: Implications for instruction of the stage theory. Journal of Developmental Education, 20 (3), 34-35. 